\title{
Communication strategies for scientific dissemination. The mUNISS Historical Scientific Museum
}

\author{
1 \\ Marco Sironi , Alfredo Calosci and Nicolò Ceccarelli \\ 1 \\ Laboratorio AnimazioneDesign, DADU, Università di Sassari, Italy \\ marcosironi@elitradesign.it, alfredoc@negot.net, nceccare@uniss.it
}

\begin{abstract}
The evolution of multimedia formats and the appearance of an ecosystem of open source solutions for digital communication promotes a process in which the construction of knowledge, once confined to very specific institutions, opens up to the contributions from a variety of new actors. A recent dissemination project for our University's Historical Scientific Museum has allowed us to confront with this emerging scenario.

This paper presents the strategies we adopted for empowering the visitor's experience to a small exhibition space intended as an introductory step to the scientific collections of the University of Sassari. The project may be considered as an attempt to communicate complexity, exploring different ways for disseminating contents, tailoring goals, targeting audiences, to a very specific context and set of resources, in the direction of developing an integrated framework of tangible and digital elements from a locally based cosmopolitan perspective.
\end{abstract}

Keywords: Exhibit Design, Dissemination of Knowledge, Transmediality

\section{A scientific heritage}

In the course of its long history, and especially since the early nineteenth century, the University of Sassari gathered a wealth of objects, instruments, books which although not always and necessarily rare or precious - give shape today to a vast and meaningful collection (Mattone 2010).

Originally intended for educational and research purposes, the Historical Scientific collection of the University encompasses more than 150.000 artifacts of different origin: scientific and laboratory equipment, mineral samples, botanical models, biological, zoological, anatomical samples and so on. Preserved as the legacy of the past, this material is hosted in ten distinct departmental collections - sometimes just by inertia, more often for its historical value. 

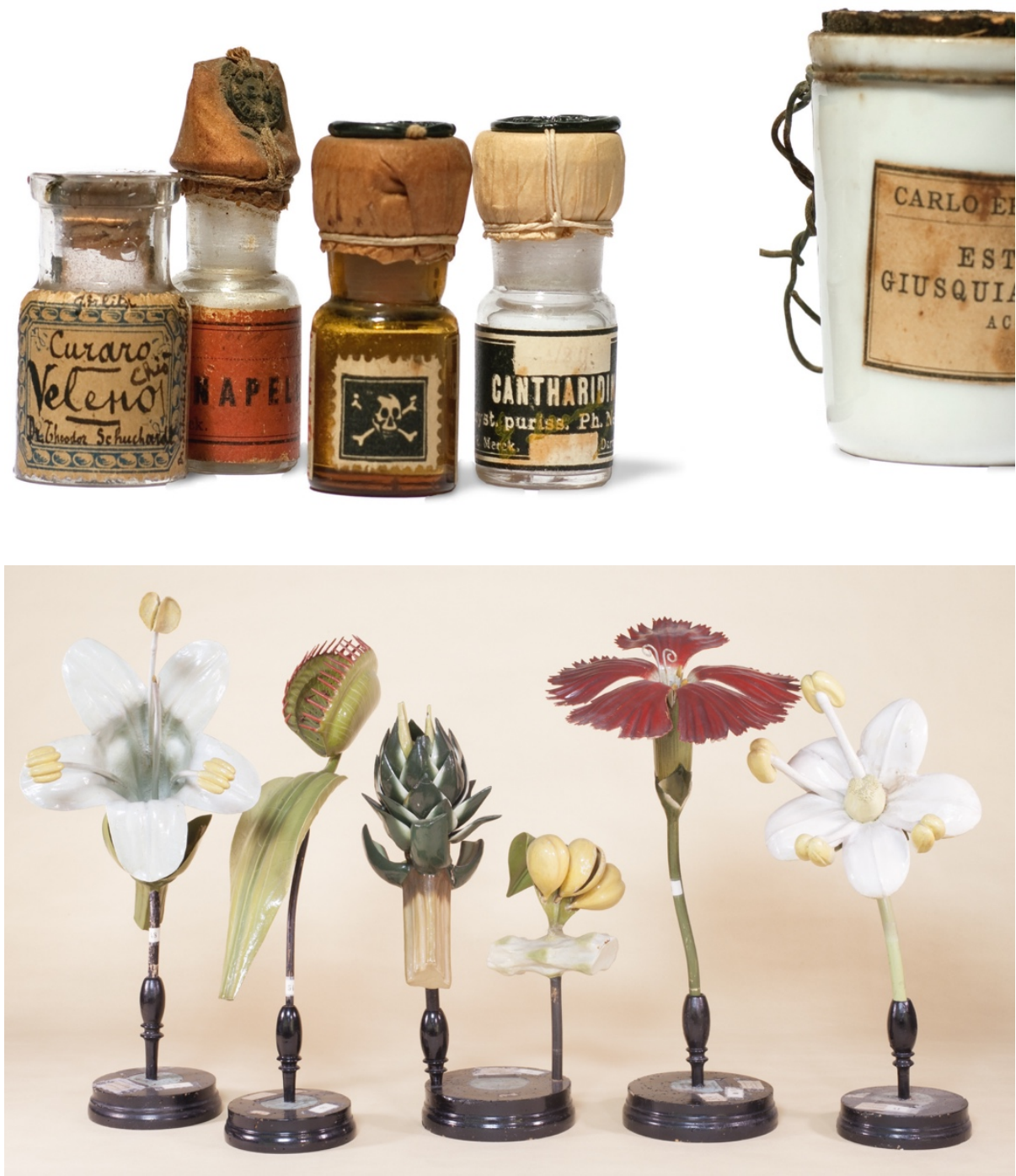

Fig. 1, 2. Selected artifacts from the museum collections: Chemistry, botanical models.

For long, the collections have been scattered in different places, hardly accessible: often unknown even to the University personnel. Under these premises, our project for the mUNISS Museum could not be reduced to a mere question of re-branding or of a signage or communication design, but needed a set of strategic solutions that could play simultaneously at several different levels. As a first step in a general valorization of the Museum collections, and as anticipation of a future re-arrangement of the collections, the University of Sassari planned to set up preliminary exhibition space, almost an introductory hub, and decided to host it in the hall of a new building that was dubbed as 'the snail' for its spiral-shaped plan. 


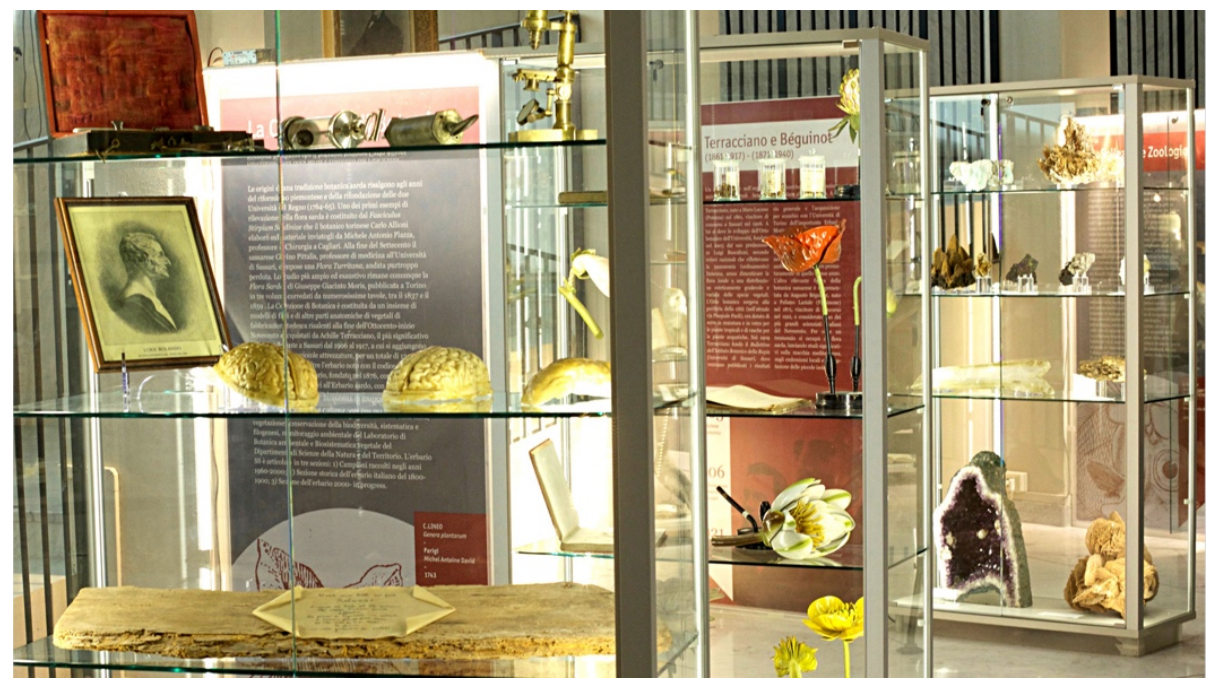

Fig. 3. Views of the exhibition space.

\section{Displaying and connecting a collection}

The very first step was improving the public's accessibility. Despite the limited set of resources - both in terms of available space and budget - we soon realized that arranging the exhibition space with curiosities from the past would not suffice, per se, to bringing the collection back to light.

By definition, a culture - and thereby cultural heritage - cannot exist if it is not shared by a community. Objects on display have very little social value unless they are culturally accessible and if they are not related to a community (Magatti 2009).

The large part of the collections is composed of scientific assets, technical equipment, texts or documents that students, researchers and lecturers would use in their everyday academics activities. The driving idea behind the project became hence reconnecting the collections to a new community of interest, inside and outside the University. In our vision, the most effective way to get in touch with a local audience was by providing a broad frame of reference, a wider perspective that would allow a better understanding of the musealia's cultural value.

A further key element in this strategy was to raise the awareness of the collection's value as a whole, among the UNISS's academic community so to gain its support, even its complicity, for the task ahead. Regardless of its relatively small size, the mUNISS collection can, as an academic museum, be considered part of a global network of scientific heritage. Its existence is testimony, thanks to the University of Sassari, a wide area in the north of Sardinia had long actively belonged to the scientific community since the very beginning.

In this framework, the inner meaning of the dissemination effort connected with the launch of the mUNISS exhibit hub is to connect the "objects on display" with their 
real life context. This may allow the general public to relate with its own conceptual references to this set of artifacts assuming - by doing so - ownership over them as commons goods, part of a cultural heritage. In order to engage this new potential audience, a cultural gap had to be filled, so the approach we decided to take was to exploit the languages and communication means our public is most used to. The project hence moves from the basic selection of meaningful artifacts that are hosted in the exhibition space and plays on the intersection between their physical dimension with the digital one, shaping a hybrid digital library of multimedia artifacts that are part of a wider inter-connected network. A network based on the idea of a variety of contact points capable of engaging a diversified audience. Another key aspect of our project, such a modular approach represents an open and scalable initiative; an agile approach allowing the entire project to grow by small incremental steps.

\section{Exhibition spaces: beyond the "snail"}

The "snail" exhibit space is intended as a first exhibition core, the main hub whose main aim is offering a general introduction to the collections originally hosted in different University departments. The snail becomes hence the place where a

From the collections to the exhibition space... and viceversa.

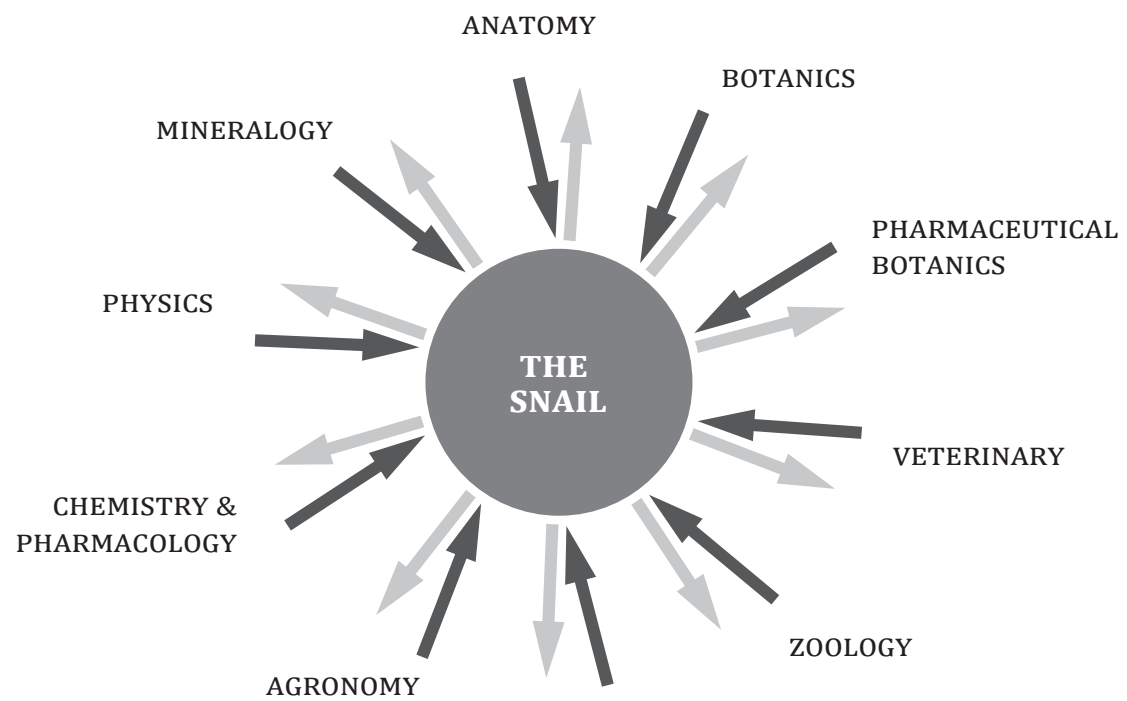

ENTOMOLOGY

Fig. 4. Functional diagrams for the "snail" hub. 
representative sample of University scientific heritage is presented, offering to visitors an anthological vision of the collections: a meaningful "cross-section" of the whole system. The displays have temporary character, and may be periodically re-arranged either for achieving a diversified offer or according to conservation matters.

The main goal of this first exhibit is to stimulate the public's curiosity towards further insights. Metaphorically speaking, we may think of it as a centrifugal gesture - directing the visitor's attention from a core exhibit to the places where the collections are actually hosted - as reaction to an opposite 'convergent' movement that attracts both users and items to the snail exhibition space.

Given its status of tangible core and contact point between the University collections as a whole and the public, the 'snail' exhibition space becomes the focal
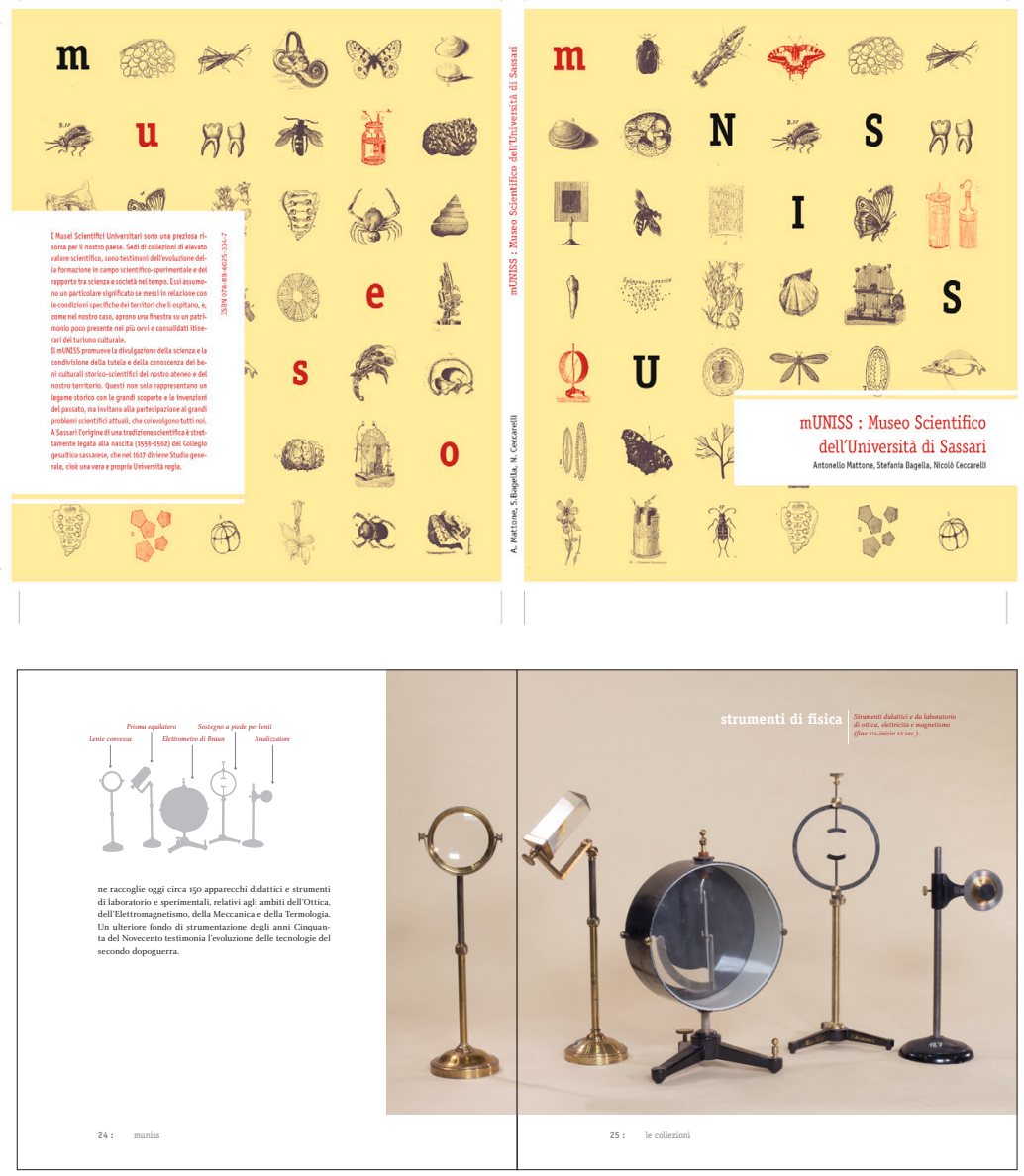

Fig. 5. Examples from the Museum's catalogue: cover and spread page. 
point for a set of communication artifacts we developed during the project: a printed catalogue, a web site and several multimedia artifacts. The development of a strong visual identity and of a system of graphics guidelines played an important role in this perspective, as they provided consistency to every communication element exhibition panels, captions, references - allowing to convey a solid identity for the Museum in every aspect of its contact with its users.

For what concerns the digital component of the project, the website we created to support the Museum's visit is conceived as a symmetrical - delocalized - hub reflecting in many ways the actual installations. Shaped on a simple structure, and divided into clearly navigable sections, the site combines practical information about

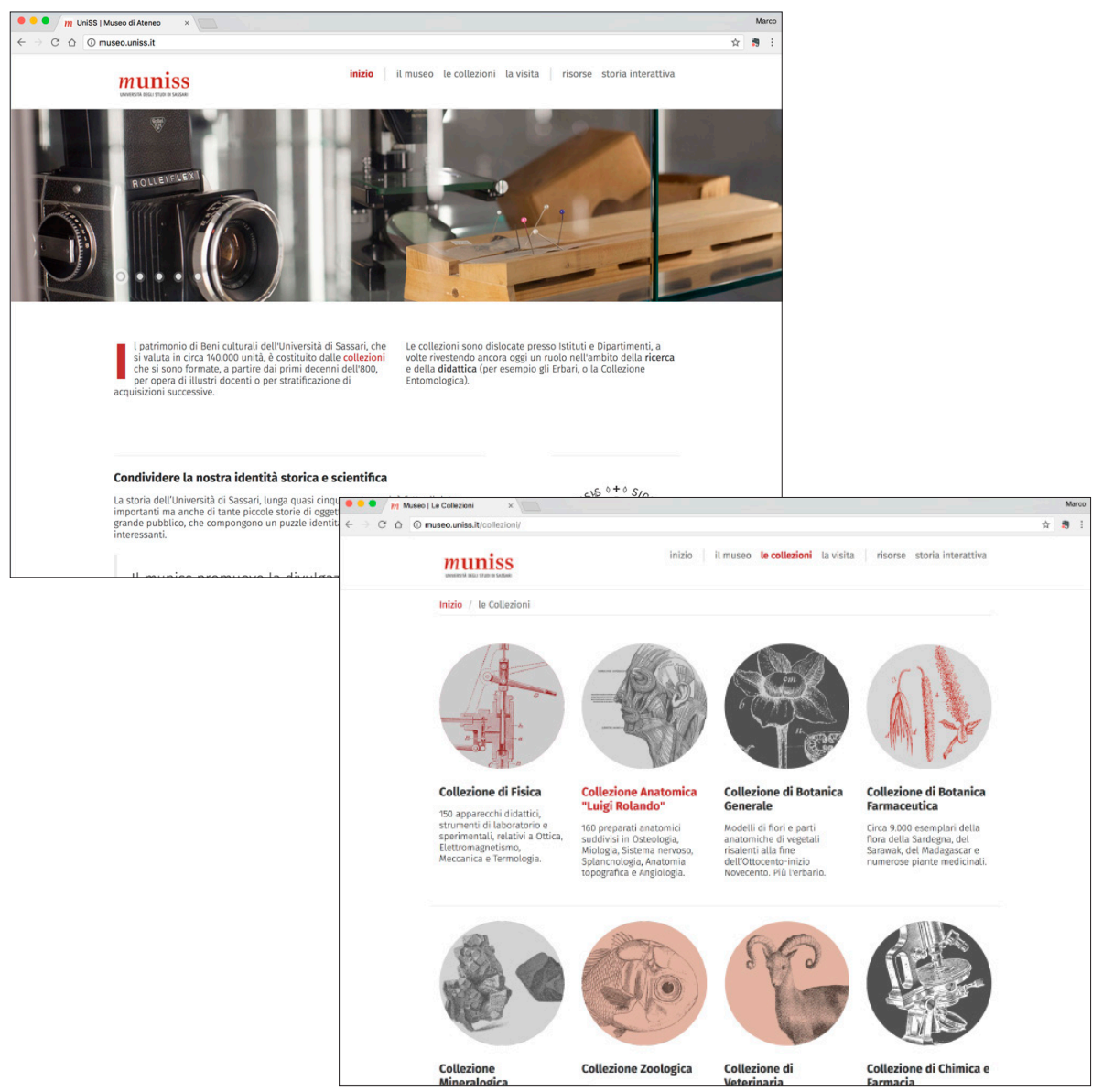

Fig. 6. Home page and Collection index from the website. 
each sub-collection. Its contents aimed at channeling the public's initial fascination risen by the historical artifacts -towards a more comprehensive understanding. This is achieved by relating a meaningful selection of the collection items with science's evolution, history and culture with an appropriate interpretative grid.

A key component of this part of the project is an interactive timeline which is intended either as a tool for on-line visitors and as an aid to run on-site multimedia interactive presentations to the collection. The key objective of this arrangement is to present a conceptual framework representing both the temporal transformations occurred in the relationship between scientific research, culture, politic and everyday life, and how a relatively marginal and peripheral university like Sassari was in fact part of a wider cultural and scientific network.

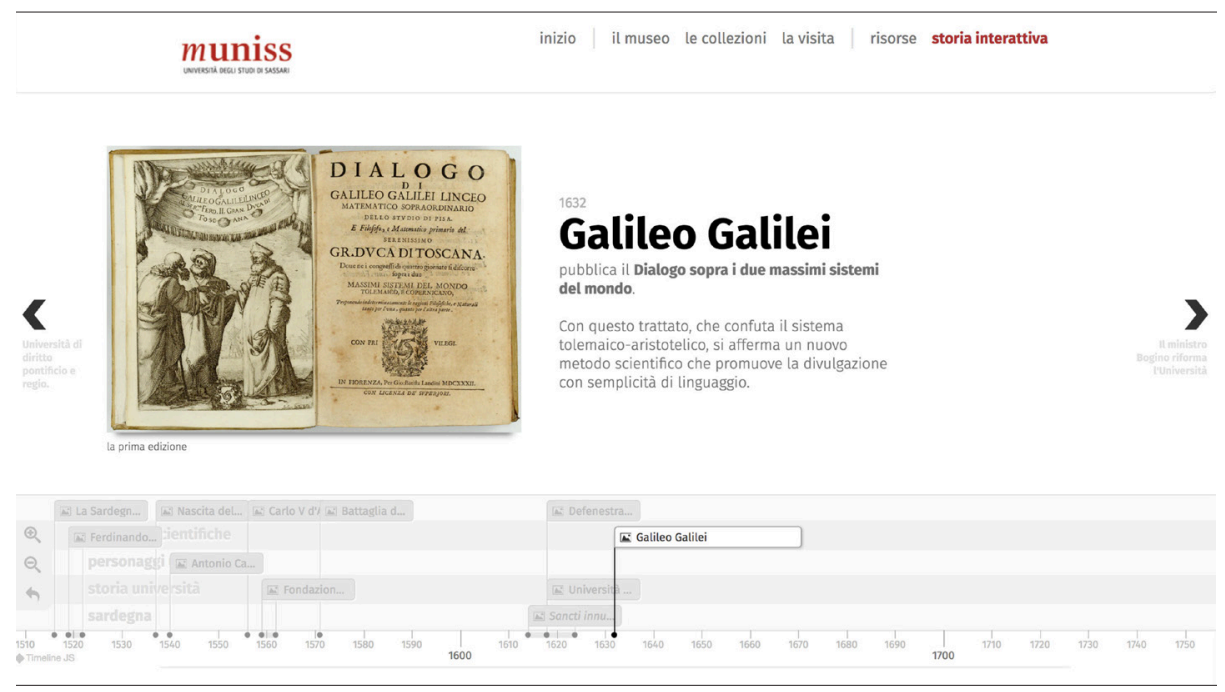

Fig. 7. mUNISS interactive timeline.

This specific objective was sought after by combining the evolution of scientific thought, some historical milestones and a selection of well-known UNISS researchers and of events or taking place in the specific local environment of Sassari and North Sardinia. Based on the open source model developed by North-Western University's Knight Laboratory ${ }^{1}$, the timeline was launched with a basic informative structure, developed under our supervision, and was opened to contributions either by other scholars (either from our university or not) as well as from the general public.

Opening the informative base already available to forms of 'external' contributions, takes a significant role in our digital dissemination strategy. In our vision, every item

1 https://knightlab.northwestern.edu 
belonging to the collections represents an opportunity, a starting point, to investigate further on the scientific topic it was originally designed for. In fact, while scientific instruments are due to become obsolete in time, a large part of the scientific topics maintain their interest and relevance, and evolve with new hypothesis and alternatives explanations.

The website hosts a small library of audiovisual modules that we created thanks for a research grant from the Italian Ministry for University. This first critical mass is intended as a prototype, a starting point to be expanded in the future. The artifacts we created for this section of the website where developed as first elements of three possible series:

1. the "Introduction to the collections" series provides an overview of two (entomology, mineralogy) of the museum main collections. Some of the highlights of each collection showcased and some information about the collection origin and history are also presented.

2. the "Book tales" series is intended as a tribute to the role of printed books in the dissemination of knowledge. The short audiovisual modules we created, present two 19th century volumes (Gray's anatomy, by H. Gray and H. Vandyke Carter, and the Ichonographia by A. Fiori), selected from the University Library's historical collections.

3. The "Experiences" series where developed as multimedia aids to demonstrate the functioning of a selection of the $19^{\text {th }}$ Century historical instruments from the Physics collection. The audiovisuals document the actual experimental conditions, recreated with the assistant of an expert and integrated by 3D and computer visualizations we expressly developed for the project.

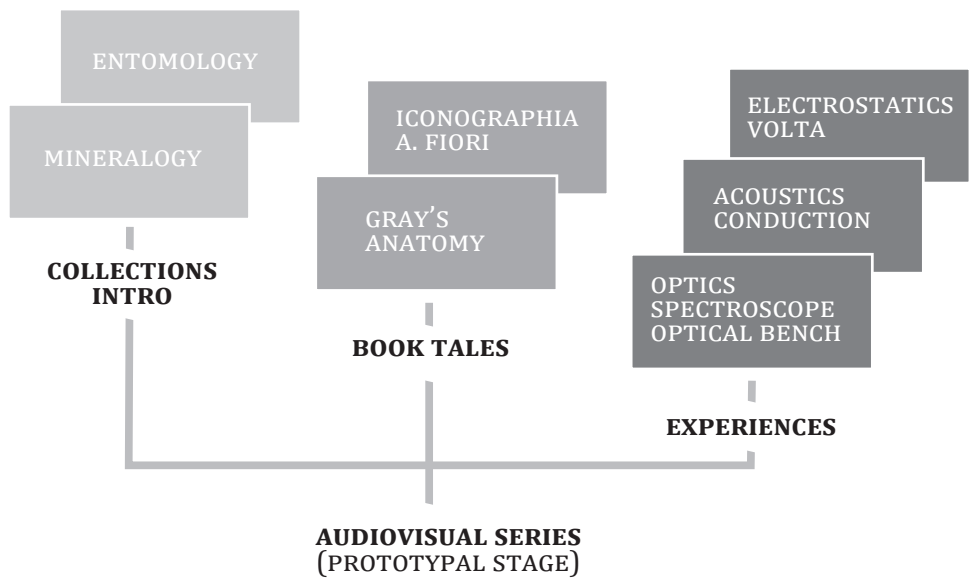

Fig. 8. The audiovisual modules hosted in the digital library. 
The audiovisual modules have been thought as learning tools, to be experienced either independently by individual users or in a guided environment, such as a classroom. The modules were developed and made available hoping they would be shared and distributed through social networks.

The idea behind their creation was in fact that, once on-line, they could become part of a wider network of scientific open-access contents. In this perspective, the informative artifacts we developed for the project represent - following the path of others institutions like the Gabinetto di Fisica of the University of Florence ${ }^{2}$ or the $\mathrm{MIT}^{3}$ - our humble contribution to a new digital scenario in which "Massive Open Online Course" (MOOC) and others less structured contents contribute in reshaping the landscape of formal and informal education.
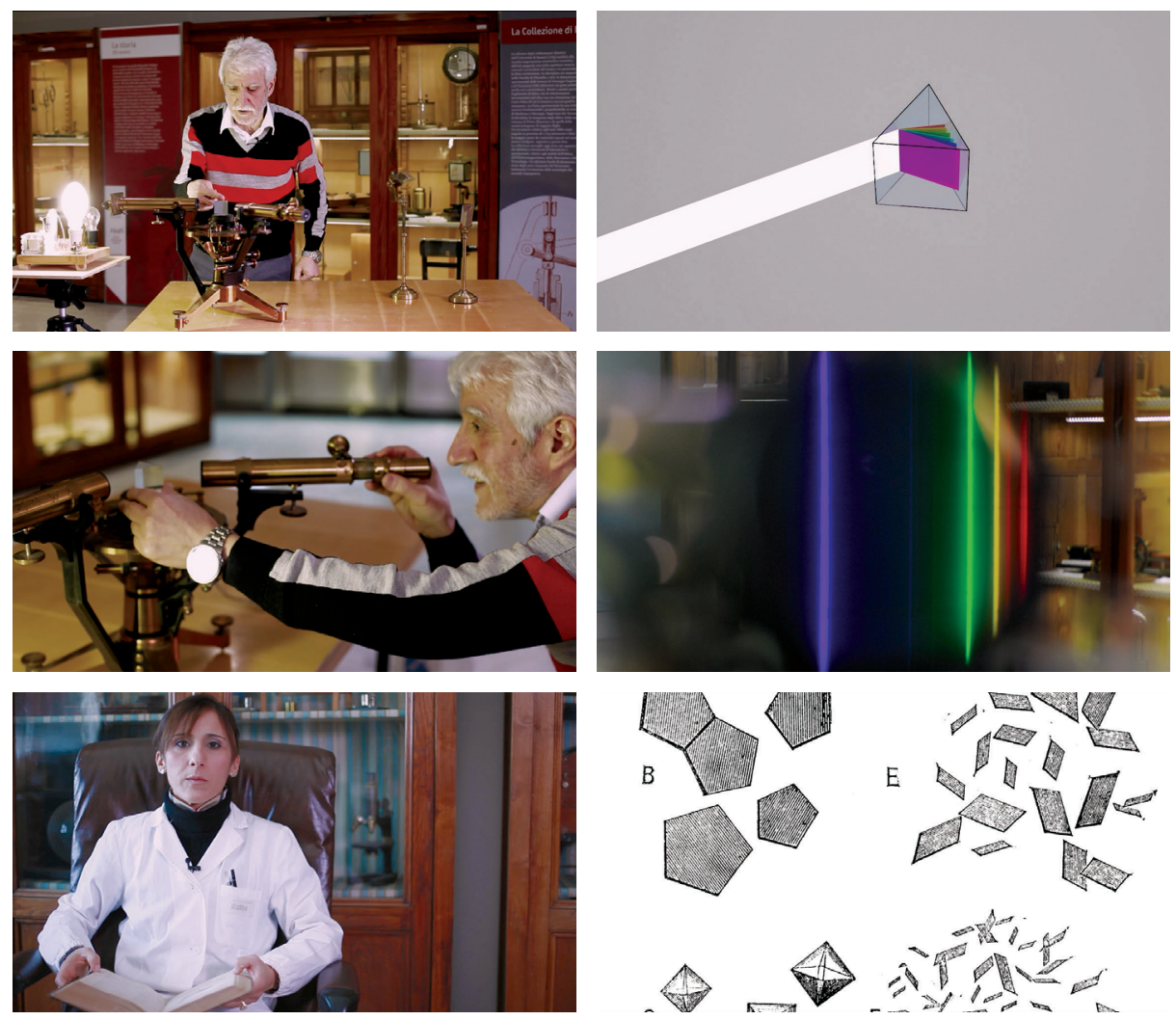

Fig. 9. Frames from the audiovisual artifacts hosted in the digital library.

\footnotetext{
${ }^{2}$ Università di Firenze - Gabinetto di Fisica: http://www.fstfirenze.it/gabinetto-di-fisica/

${ }^{3}$ MIT Open Courseware: https://ocw.mit.edu/
} 
The direct involvement of various members of the academic community as experts, consultant, co-creators and - often - even as presenters in front of the camera, was a great opportunity for consolidating a community of interest around the museum's activities. The idea of involving the university academic body in the development and production of such audiovisual contents was instrumental to stimulating the local community in having a part in the new museum project.

Promoting such an involvement, we believe, ought to be considered a main asset for any kind of cultural enterprise, in terms of developing a fruitful and dialectic interaction with its public. On the other side, speaking in strictly technical terms, although possibly more effective in productive terms, resorting to professional speaker as presenter would have not been as effective as the presence of a member of the actual community.

Digital media represents a great opportunity to enhance the way heritage information can be delivered to a museum's public, at the many different stages of their complex relationship. Online-contents can be used before the visit, during its planning, or after that, as an opportunity for recall to memory or for further inquiry. And of course they can, to some extent, substitute the visit itself. Nevertheless, in our vision for the mUNISS project, the coexistence of both the physical and digital dimensions does provide the opportunity of orchestrating a more comprehensive experience.

\section{PHISICAL SPACE}

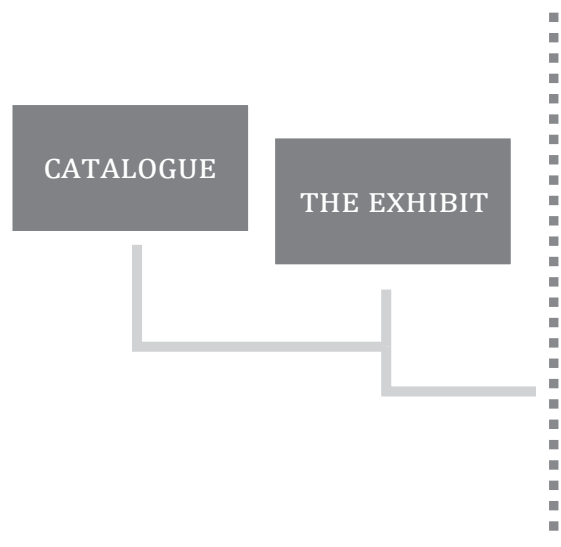

VIRTUAL SPACE

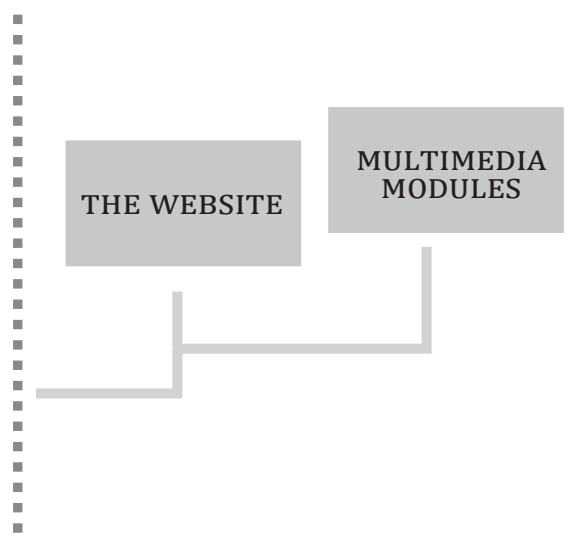

Fig. 10. Having the physical and virtual dimensions interplay. 


\section{A systemic approach}

The reference model for our project is a contemporary and multidisciplinary systemic approach based on a strategy in which a complex "multi layered" system of informative elements gives shape to a peculiar approach to communication. Designers are increasingly (Anceschi 2003) taking the role of "directors" or "coordinators" rather than of "creators". An essential requirement for pursuing this approach is to foster a fluid dialog among the various stakeholders involved in the project. Such a dialogic attitude, today a frequent component of design practices, has strong roots in the tradition of Italian design. This is the case, for instance, of the work carried out in the 50's and 60's by some well-known authors. In Albe Steiner's or Roberto Sambonet's graphic work - that anticipated today's generative approach to logos and monograms, for instance, (Camuffo et al. 2012) or in the exhibit design projects by Castiglioni, Tovaglia and Huber, and noticeably in the "comprehensive" and "integrated" designs by Achille and Pier Giacomo Castiglioni (Migliore et al. 2018).

\section{Conclusions}

The strategic criteria upon which the mUNISS project was developed are inherently systemic, as they take advantage from the active intersections between various design disciplines, underlining their common roots and tracing new areas of application in the domain of communicating complexity.

Our approach starts by giving up any methodological shortcuts or off the shelf solutions. In this approach, basic preliminary research is carried out to generate a "conceptual cartography" and letting, once established the final goal/destination of our design journey, the project 'find its own way to its solution' - approaching the final goal by a progressive fine tuning process. Along this eventful path, different skills and design elements of knowledge need to be orchestrated and combined in order to converge towards to a common integrated design proposal.

By operating strategically, communication design can efficiently contribute to the diffusion of knowledge. It can promote its dissemination by helping - new and old public institutions playing their role in the contemporary scenario of global and digital communication networks.

In mUNISS' project key role is played by the contribution of a wide area of potential users-actors (Manzini 2015), urged to take action, often by sharing their knowledge and their passionate relationship with the collections and with the artifacts on display. In our vision, this is a key step towards sowing the seeds for the birth of a first nucleus of a new community. 


\section{References}

Anceschi, G. (2003, June). Progetto grafico. Progetto Grafico, n. 1, p. 3.

Camuffo, G., Piazza, M., Vinti, C., Annicchiarico S. (Eds). (2012). TDM 5: Grafica italiana. Mantova : Corraini.

Lorenc J. (2007). What is exhibition design? London, Rotovision.

Magatti, M., (2009), Libertà immaginaria. Le illusioni del capitalismo tecno-nichilista, Feltrinelli : Milano.

Manzini, E. (2015). Design when Everybody Designs. An Introduction to Design for Social Innovation. Cambridge, MA : the MIT Press.

Mattone, A. (2010), Storia dell'Università di Sassari, 1, Nuoro : Illisso Edizioni.

Migliore, I., Servetto, M., Lupi, I., Ossanna Cavadini, N. (Eds). (2018). Achille Castiglioni visionary. The installational alphabet of a designer director. Milano : Skira.

Università di Firenze - Gabinetto di Fisica: http://www.fstfirenze.it/gabinetto-di-fisica/ MIT Open Courseware: https://ocw.mit.edu/ 